\title{
"Tupanvirus", a new genus in the family Mimiviridae
}

\author{
Rodrigo Araújo Lima Rodrigues ${ }^{1,2} \cdot$ Said Mougari ${ }^{1} \cdot$ Phillipe Colson $^{1} \cdot$ Bernard La Scola ${ }^{1}$ Jônatas Santos Abrahão ${ }^{2}$ (I)
}

Received: 6 July 2018 / Accepted: 30 September 2018 / Published online: 5 October 2018

(c) Springer-Verlag GmbH Austria, part of Springer Nature 2018

\begin{abstract}
The genus "Tupanvirus" is a new proposed taxon to be included in the family Mimiviridae. The two known tupanvirus isolates were isolated from soda lake and oceanic sediments samples collected in Brazil and were named "tupanvirus soda lake" and "tupanvirus deep ocean", respectively. These viruses exhibit similarities to amoeba-infecting mimiviruses, but there are also several differences that place them in a separate group within the family Mimiviridae. Their virions have a mean size of $1.2 \mu \mathrm{m}$, which include a mimivirus-like capsid and a large cylindrical tail, both covered by fibrils. The linear double-stranded DNA genomes of up to 1,516,267 base pairs encode over 1,200 genes, among which 30\% have no homologs in any database, including in other mimivirus genomes. Compared to other mimiviruses, tupanviruses exhibit a broader host range and cause a cytotoxic effect in host and non-host organisms, a phenotype that is not observed for other mimiviruses. Remarkably, these viruses possess the most complete gene set related to the protein synthesis process, including 20 aminoacyl-tRNA synthetases, 67-70 tRNAs, many translation factors, and genes involved in maturation and modification of tRNA and mRNA, among others. Moreover, diverse phylogenomic analyses put tupanviruses in a distinct group within the family Mimiviridae. In light of the set of different features observed for these giant viruses, we propose establishment of a new genus to allow proper classification of two known tupanviruses and possibly many more similar viruses yet to be characterized.
\end{abstract}

\section{Introduction}

The discovery of Acanthamoeba polyphaga mimivirus (APMV) in 2003 paved the way for new and outstanding discoveries in the virology field, expanding our knowledge about diversity, evolution and complexity of viruses [1]. Given to its distinct morphological and genetic features, a new viral family, named Mimiviridae, was created to

Handling Editor: Sead Sabanadzovic.

Rodrigo Araújo Lima Rodrigues and Said Mougari contributed equally to this work.

The taxonomic changes proposed here have not been endorsed by the ICTV Executive Committee. This article is related to an ongoing taxonomic proposal, submitted to the ICTV but not yet accepted at the time of submission.

Jônatas Santos Abrahão

jonatas.abrahao@gmail.com

1 Aix-Marseille Université, IRD, APHM, MEPHI, IHUMéditerranée Infection, Marseille, France

2 Laboratório de Vírus, Departamento de Microbiologia, Instituto de Ciências Biológicas, Universidade Federal de Minas Gerais, Belo Horizonte, Minas Gerais, Brazil accommodate this virus [2]. This family currently includes two genera, the genus Mimivirus, in which the only officially recognized species is typified by APMV, and the genus Cafeteriavirus, with a single species, Cafeteria roenbergensis virus, whose members are distantly related to APMV and infect marine flagellates [3].

Dozens of other mimiviruses infecting free-living amoebae have been described over the past decade in different parts of the world and from distinct environments/hosts, and these viruses exhibit biological, structural and genomic characteristics similar to those observed for APMV [4-11]. In addition, some viruses that are known to infect unicellular algae are proposed members of the family Mimiviridae, since they are phylogenetically closer to amoebae-infecting mimiviruses than to other algae-infecting viruses, which are usually classified in the family Phycodnaviridae [12]. Furthermore, a new virus infecting a kinetoplastid protozoan, named "Bodo saltans virus" [13], was recently proposed to be part of a new group of complex mimiviruses, along with four other viruses whose genomes were assembled from environmental metagenomes but that were not isolated, and these were proposed to comprise a subfamily named "Klosneuvirinae" within the family Mimiviridae [14]. 
Recently, we described the discovery of two new members of the family Mimiviridae, the tupanviruses, which have a set of features that strongly differ from the mimiviruses described to date [15]. These viruses were isolated from soda lake water and oceanic sediment samples collected in Brazil and were named in tribute to Tupan - or Tupã - (God of Thunder) which is an important mythological figure to the South American Guarani indigenous tribes. The new and intriguing characteristics observed for the tupanviruses led us to propose the creation of a new taxon in the family Mimiviridae, the genus "Tupanvirus".

\section{Morphological properties}

The tupanviruses have a capsid similar in size to those observed for other mimiviruses $(\sim 450 \mathrm{~nm})$, which exhibit a 'stargate' structure [16] and is covered by fibrils. However, unlike other viruses, the tupanviruses have a large cylindrical tail attached to the base of the capsid of $\sim 550 \mathrm{~nm}$ in length and $\sim 450 \mathrm{~nm}$ in diameter (including fibrils) (Fig. 1A and B). The mean size of tupanvirus' virions is $1.2 \mu \mathrm{m}$, which allows them to be visualized easily using optical microscopy, although particles up to $2.3 \mu \mathrm{m}$ have been observed due to a high degree of plasticity in size of the tail [15]. A lipid membrane is present in the capsid, similar to other giant viruses $[17,18]$, which is associated with fusion with the
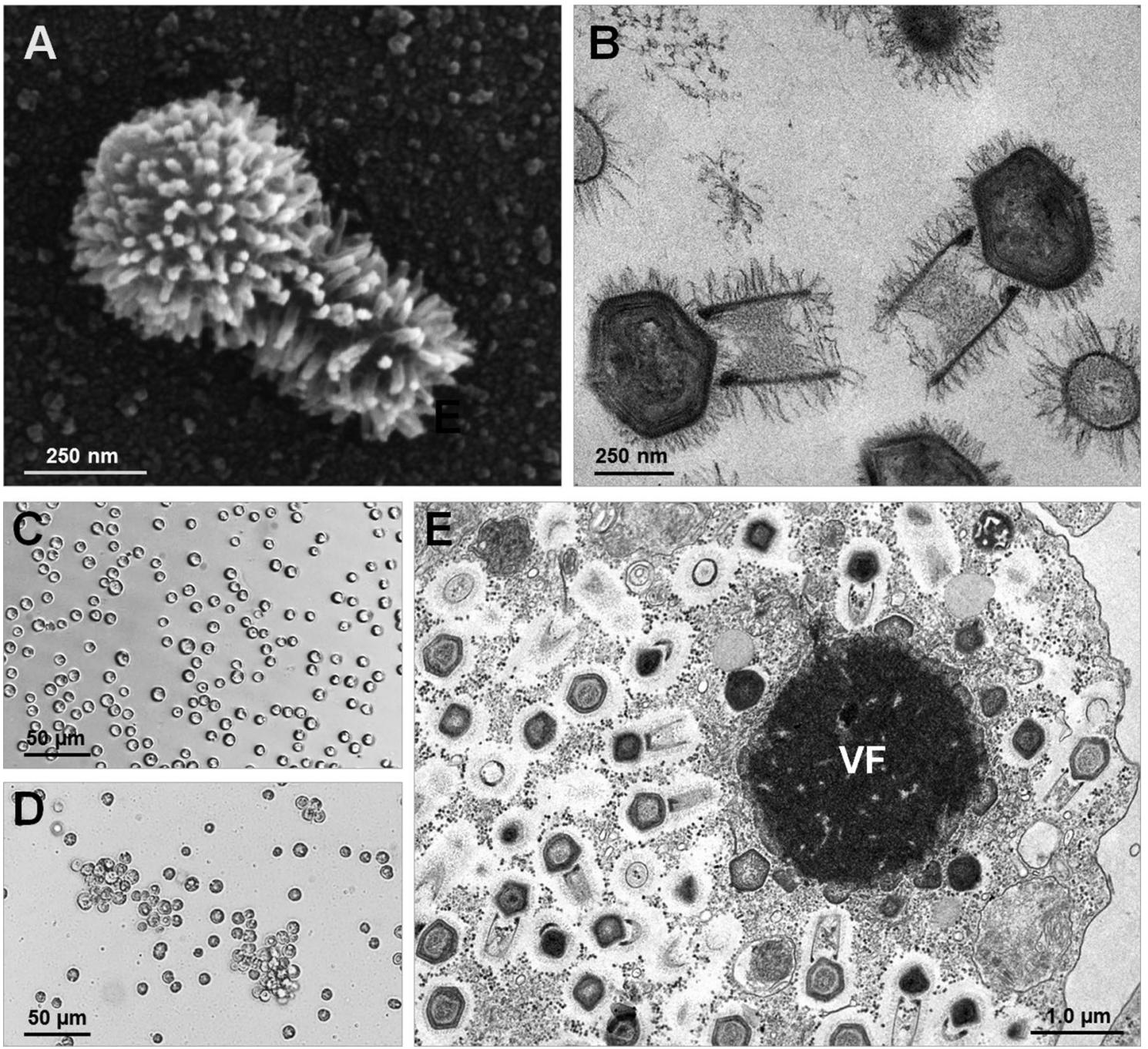

Fig. 1 Morphological and replication properties of tupanviruses. (A) Scanning electron microscopy of a tupanvirus particle. (B) Transmission electron microscopy (TEM) of tupanvirus particles, showing a mimivirus-like capsid and a cylindrical tail attached at its base. Opti- cal microscopy of cytopathic effect of mimivirus (C) and tupanvirus (D). (E) TEM image of an A. castellanii cell infected with a tupanvirus, representing a late stage of the replication cycle, showing the viral factory (VF) and mature viruses around this structure 
phagosome membrane and release of the capsid contents. The tail is less electron-dense than the capsid and its contents are still unknown (Fig. 1B). Nevertheless, this material seems to be released into the host cytoplasm after invagination of the phagosome membrane inside the tail [15].

\section{Prevalence and host range}

The distribution of tupanviruses in nature is uncertain. The two viruses were isolated from separate samples and had different characteristics. The first one was isolated from water samples collected from Brazilian soda lakes, which have high salinity and $\mathrm{pH}$ ( 9.0), and it was named "tupanvirus soda lake" (TPV-SL), while the second one, named "tupanvirus deep ocean" (TPV-DO), was isolated from ocean sediment samples collected at a depth of $3000 \mathrm{~m}$ in the Brazilian Atlantic Ocean (Table 1). Interestingly, a mimivirus-like endoparasite with a thin tail attached to the capsid was isolated from plane tree bark samples collected in Germany using Saccamoeba sp. as bait, and was named KSL5x [19]. Since no genomic information is available about KSL5x, it is not possible to confirm that it is another tupanvirus, although they are morphologically similar. New analysis should be conducted in this regard, which could provide valuable insights about the ecology and evolution of these viruses.

The natural hosts of tupanviruses are also unknown. Unlike other amoeba-infecting mimiviruses, the tupanviruses are able to infect and establish a productive cycle in vitro in different species of amoebae of the genus Acanthamoeba, Vermamoeba vermiformis, Dictyostelium discoideum and Willartia magna [15]. Furthermore, a cytotoxic phenotype in non-host cells (Tetrahymena hyperangularis, RAW247, and THP-1 cells) was observed, as well as for $A$. castellanii at high multiplicity of infection, something that is not observed for APMV. This toxic profile seems to be related to a shutdown of ribosomal RNA in infected cells, while the autophagy/ribophagy canonical pathways are not involved in the process, and thus the mechanism of this phenotype is still unknown [15].

\section{Properties in culture}

The tupanviruses cause a cytopathic effect in A. castellanii cells that is typical of mimiviruses, characterized by loss of motility, rounding, and further cell lysis. However, unlike what is usually observed for mimiviruses, the tupanviruses caused cell aggregation, forming clusters of round cells previous to the lysis step (Fig. 1C and D). At the ultrastructural level, the tupanviruses display a replicative cycle similar to that of other mimiviruses $[15,20]$. Viral particles enter into host cells by phagocytosis $1 \mathrm{~h}$ postinfection. After opening 
of the 'stargate' and fusion of capsid inner membrane to the phagosome membrane, the genome is released to the host cytoplasm, where a viral factory is established (Fig. 1E). In this region, genome replication and viral morphogenesis takes place; the capsid is built first and then the tail is attached. Mature virions are released by cell lysis in the last step of the viral cycle.

\section{Genomic and proteomic features}

The genomes of tupanviruses are linear double-stranded DNA molecules with $~ 28 \% \mathrm{G}+\mathrm{C}$. The TPV-SL genome is $1,439,508 \mathrm{bp}$ in length and contains 1276 genes, while the TPV-DO has a genome of $1,516,267 \mathrm{bp}$ that contains 1359 genes (Table 1). Around $30 \%$ of the predicted genes for the tupanviruses are completely new, named ORFans (open reading frames with no similarities in the databases). Among the ORFs with known function, up to 50\% exhibit best hits in other viruses, especially members of the family Mimiviridae, while the other genes have best hits in cellular organisms, mainly eukaryotes and bacteria [15]. The structure and genome architecture are well conserved between the two tupanviruses, except for the terminal region of the genome of TPV-SL, which appears to be translocated and inverted in the genome of TPV-DO. In contrast, these genomes exhibit a strongly different synteny when compared to the genomes of the prototype viruses of the genera Mimivirus and Cafeteriavirus (Fig. 2). Moreover, the tupanviruses have the promoter motif AAAATTGA and a codon and amino acid usage frequency similar to that reported for other mimiviruses $[3,15,21]$.

The tupanviruses have the most complete arsenal of genes related to the protein translation process among the members of the virosphere. Surprisingly, these viruses encode 67-70 tRNAs, 20 aminoacyl-tRNA synthetases (aaRS), and 41-44 other proteins involved in the translation process, such as translation factors (initiation, elongation and release), and factors related to maturation of tRNA and mRNA, among others (Table 1). The amount and diversity of these genes far exceed what was previously known for other giant viruses, even including the klosneuviruses, which possess up to 19 aaRS [14], and Bodo Saltans virus. The origin of these genes is still a matter of a hot debate in the scientific community, and a consensus has not yet been reached on whether these genes came from several events of lateral gene transfer from hosts and/or sympatric organisms or were already contain in the ancestors of mimiviruses [22-24]. Furthermore, the tupanviruses present two copies of an intronic region of the 18S rRNA gene, which are highly expressed during the viral replication cycle, but their function remains to be elucidated [15]. It is noteworthy that no exonic region of the 18S rRNA gene was found in tupanviruses or other viruses, nor do they possess genes related to the energy synthesis process [15].

Proteomic analysis of TPV-SL particles revealed the presence of 127 proteins, of which 67 have no known function, and 11 are encoded by ORFans. No aaRS or translation factors were detected in the viral particles. Among the

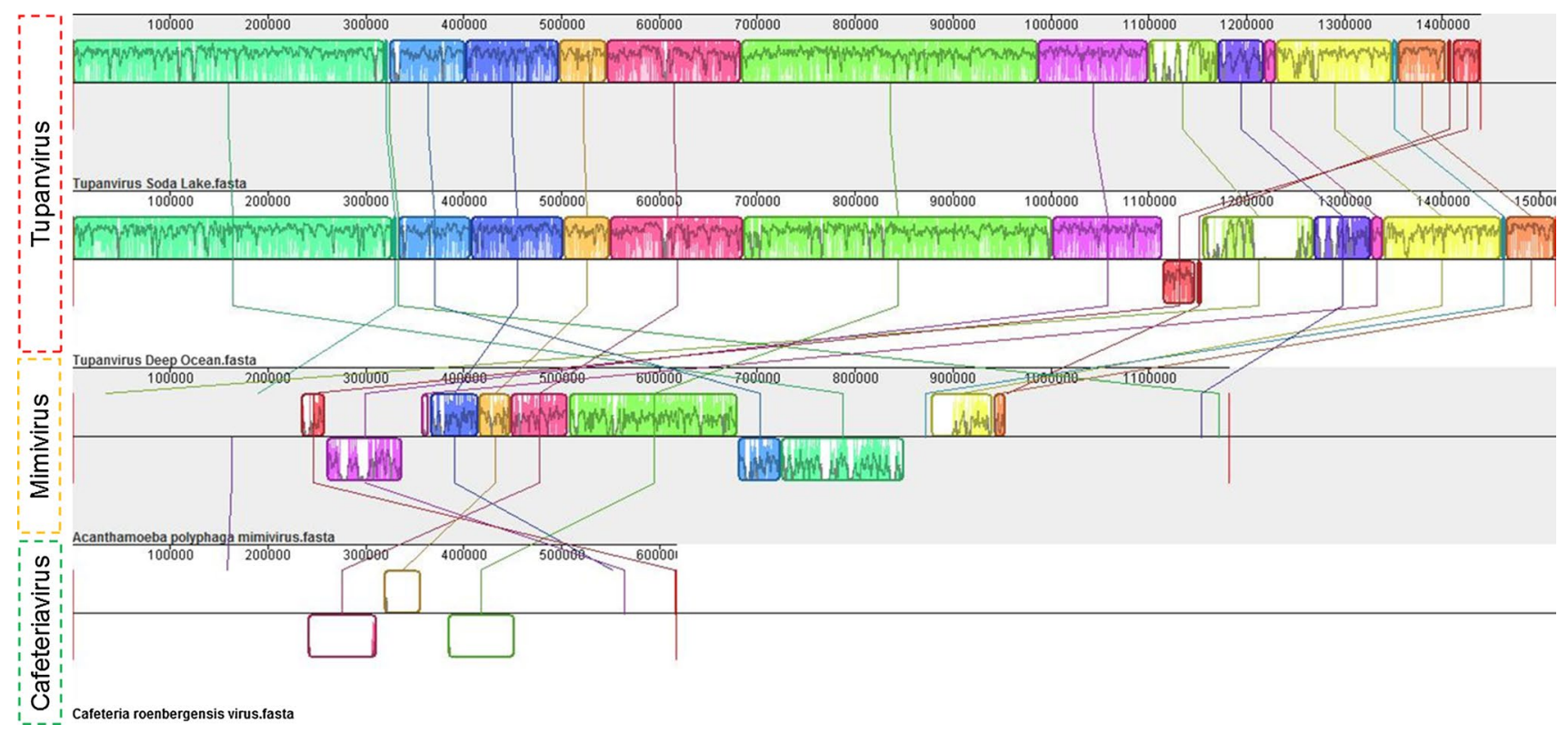

Fig. 2 Genome alignment of tupanviruses, APMV (NC_014649.1), and CroV (NC_014637.1). The figure shows genome architecture and synteny of prototype viruses of the genera of the family Mimiviridae. The boxes highlight the collinear blocks of similarity between the genomes. The graphs within the boxes represent the average similarity of each region, with a range of values and the mean value darkened. The schematic genome alignment diagram was produced using the MAUVE software package [26] 
proteins with a known function, several structural components were observed, such as the major capsid protein and the core protein, as well as enzymes involved in nucleic acid metabolism, such as a DNA polymerase, a DNA-dependent RNA polymerase, a RNA helicase, and an mRNA capping enzyme. It is notable that fewer than $50 \%$ of the proteins found in TPV-SL particles are shared with APMV or CroV [15].

\section{Phylogenomics}

Phylogenetic analysis based on different core genes of giant viruses, named DNA polymerase B family, major capsid protein, D5 primase helicase and D6/D11 helicase (both individual and concatenated) put the tupanviruses within the family Mimiviridae (Fig. 3). Notably, when the phylogenetic reconstruction is performed using the D6/D11 helicase gene, the tupanviruses are placed as a sister group of amoebaeinfecting mimiviruses (Fig. 3D). A comparable topology is observed when the analysis is carried out by hierarchical clustering based on a presence-absence matrix of clusters of orthologous genes shared by the different mimiviruses [15]. Moreover, our analyses corroborate previous studies that indicate the existence of two other groups of mimiviruses, referred to as "extended-family" Mimiviridae, comprising the algae-infecting mimiviruses and the klosneuviruses [12, 13]. These analyses highlight the differences between tupanviruses and other mimiviruses and the need to create a new taxonomic group to properly classify these new viruses.

\section{Conclusion}

The tupanviruses represent a new group of viruses isolated from extreme environments in Brazil. Although they share many features with mimiviruses of amoebae, the tupanviruses have a set of distinctive features that place them in a different group within the family Mimiviridae, the proposed genus "Tupanvirus". This new taxon would initially include
A

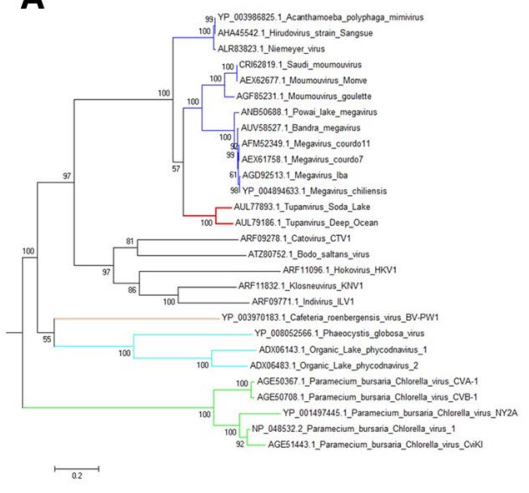

C

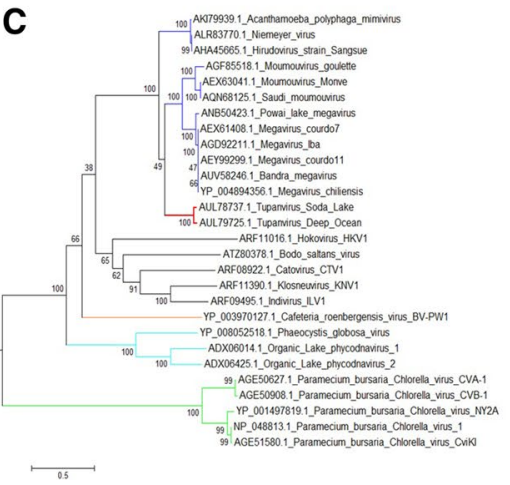

B

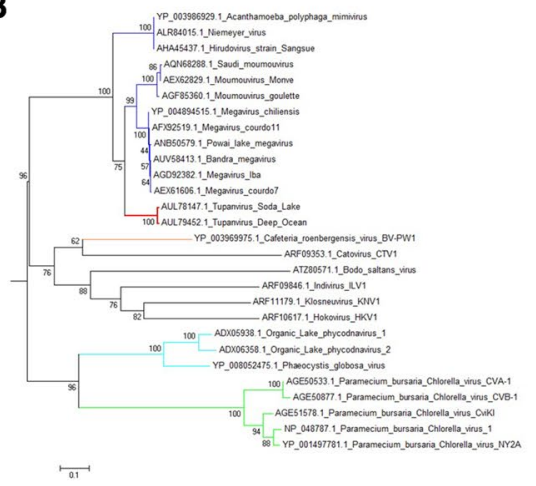

D

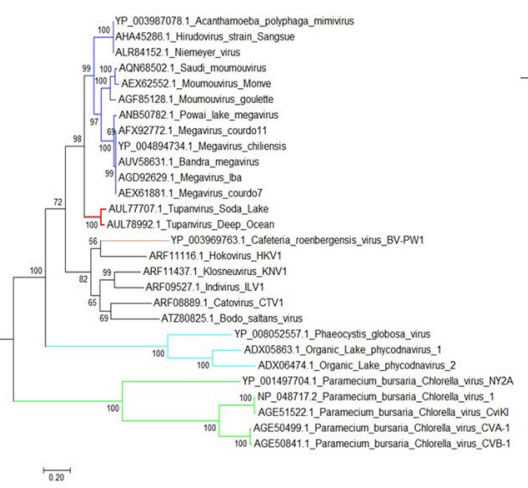

E

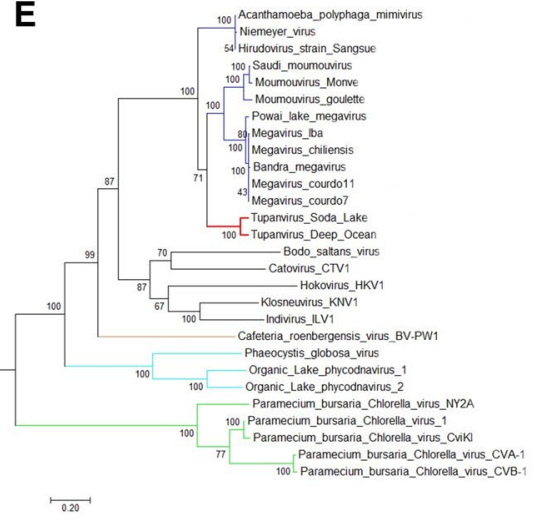

Fig. 3 Phylogenetic reconstruction using amino acid sequences of core genes of the nucleo-cytoplasmic large DNA viruses. The analyses were performed using different representatives of the family Mimiviridae and phycodnaviruses as an external group, based on (A) DNA polymerase B family, (B) major capsid protein, (C) D5 primase helicase, (D) D6/D11 helicase, and (E) the genes concatenated. The alignments were built using Muscle software [27]. The tree was con- structed using MEGA version 6.0, applying the maximum-likelihood method and JTT model of evolution with 1000 bootstrap replicates [28]. Colors indicate different viral groups: blue, mimivirus lineage $\mathrm{A}, \mathrm{B}$, and $\mathrm{C}$; red, tupanviruses; orange, CroV; black, klosneuviruses; cyan, extended Mimiviridae; green, Phycodnaviridae. Scale bars indicate rate of evolution 
two species, "Tupanvirus soda lake" and "Tupanvirus deep ocean". With the advancement of the isolation and characterization techniques of giant viruses, we can expect even more new viruses to be revealed in the near future, therefore expanding our knowledge about the virosphere.

The Mimiviridae taxonomy is an open field that requires further attention. Many mimiviruses have been described during the last few years, comprising three different clades named lineage $\mathrm{A}, \mathrm{B}$, and $\mathrm{C}$, represented by mimivirus, moumouvirus, and megavirus, respectively [4-6]. These viruses exhibit considerable differences at the genomic level that might constitute different genera, thus splitting the genus Mimivirus. Furthermore, the klosneuviruses could represent another genus within the family, alongside with the now proposed "Tupanvirus", which would be part of a subfamily, as previously suggested for the amoebae-infecting mimiviruses [25]. Additional efforts should be made to reorganize the taxonomy of the whole family Mimiviridae, which will strongly contribute to the systematics of mimiviruses.

Acknowledgements We thank colleagues from Aix Marseille University and from Universidade Federal de Minas Gerais, and the Center of Microscopy of UFMG for technical assistance. We also thank CAPES, CNPq, FAPEMIG and Mediterranée Infection for scholarships and grants. JSA is a CNPq researcher. The authors are members of a CAPES-COFECUB project. The authors declare no conflict of interest.

\section{Compliance with ethical standards}

Ethical statement We declare that this work complies with the ethical rules applicable for this journal.

\section{References}

1. Colson P, La Scola B, Levasseur A et al (2017) Mimivirus: leading the way in the discovery of giant viruses of amoebae. Nat Rev Microbiol 15:243-254. https://doi.org/10.1038/nrmicro.2016.197

2. Raoult D, Audic S, Robert C et al (2004) The 1.2-Megabase genome sequence of Mimivirus. Science 306(80):1344-1350. https://doi.org/10.1126/science.1101485

3. Fischer MG, Allen MJ, Wilson WH, Suttle CA (2010) Giant virus with a remarkable complement of genes infects marine zooplankton. Proc Natl Acad Sci 107:19508-19513. https://doi. org/10.1073/pnas.1007615107

4. Colson P, Yutinf N, Shabalina SA et al (2011) Viruses with more than 1,000 genes: Mamavirus, a new Acanthamoeba polyphaga mimivirus strain, and reannotation of mimivirus genes. Genome Biol Evol 3:737-742. https://doi.org/10.1093/gbe/evr048

5. Arslan D, Legendre M, Seltzer V et al (2011) Distant Mimivirus relative with a larger genome highlights the fundamental features of Megaviridae. Proc Natl Acad Sci 108:17486-17491. https:// doi.org/10.1073/pnas.1110889108

6. Yoosuf N, Yutin N, Colson P et al (2012) Related giant viruses in distant locations and different habitats: Acanthamoeba polyphaga moumouvirus represents a third lineage of the Mimiviridae that is close to the Megavirus lineage. Genome Biol Evol 4:1324-1330. https://doi.org/10.1093/gbe/evs109
7. Dornas FP, Khalil JYB, Pagnier I et al (2015) Isolation of new Brazilian giant viruses from environmental samples using a panel of protozoa. Front Microbiol 6:1-9. https://doi.org/10.3389/fmicb .2015 .01086

8. Saadi H, Reteno DGI, Colson P et al (2013) Shan virus: a new mimivirus isolated from the stool of a tunisian patient with pneumonia. Intervirology 56:424-429. https://doi.org/10.1159/00035 4564

9. Campos RK, Boratto PV, Assis FL et al (2014) Samba virus: a novel mimivirus from a giant rain forest, the Brazilian Amazon. Virol J 11:95. https://doi.org/10.1186/1743-422X-11-95

10. Andrade KR, Boratto PPVM, Rodrigues FP et al (2014) Oysters as hot spots for mimivirus isolation. Arch Virol 160:477-482. https ://doi.org/10.1007/s00705-014-2257-2

11. Andrade ACDSP, Arantes TS, Rodrigues RAL et al (2018) Ubiquitous giants: a plethora of giant viruses found in Brazil and Antarctica. Virol J 15:1-10. https://doi.org/10.1186/s1298 5-018-0930-x

12. Santini S, Jeudy S, Bartoli J et al (2013) Genome of Phaeocystis globosa virus PgV-16T highlights the common ancestry of the largest known DNA viruses infecting eukaryotes. Proc Natl Acad Sci USA 110:10800-10805. https://doi.org/10.1073/pnas.13032 51110

13. Deeg CM, Chow C-ET, Suttle CA (2018) The kinetoplastid-infecting Bodo saltans virus (BsV), a window into the most abundant giant viruses in the sea. Elife. https://doi.org/10.7554/elife.33014

14. Schulz F, Yutin N, Ivanova NN et al (2017) Giant viruses with an expanded complement of translation system components. Science 356(80):82-85. https://doi.org/10.1126/science.aal4657

15. Abrahão J, Silva L, Silva LS et al (2018) Tailed giant Tupanvirus possesses the most complete translational apparatus of the known virosphere. Nat Commun 9:749. https://doi.org/10.1038/s4146 7-018-03168-1

16. Zauberman N, Mutsafi Y, Halevy DB et al (2008) Distinct DNA exit and packaging portals in the virus Acanthamoeba polyphaga mimivirus. PLoS Biol 6:1104-1114. https://doi.org/10.1371/journ al.pbio.0060114

17. Mutsafi Y, Shimoni E, Shimon A, Minsky A (2013) Membrane assembly during the infection cycle of the giant mimivirus. PLoS Pathog 9:100. https://doi.org/10.1371/journal.ppat.1003367

18. Mutsafi Y, Fridmann-Sirkis Y, Milrot E et al (2014) Infection cycles of large DNA viruses: emerging themes and underlying questions. Virology 466-467:3-14. https://doi.org/10.1016/j.virol .2014.05.037

19. Michel R, Loch S, Muller KD et al (2017) Reisolation of mimivirus-like giant viruses by using Saccamoeba sp. as bait resulted in loss of virophages. Endocytobiosis Cell Res 28:50-53

20. Andrade ACDSP, Rodrigues RAL, Oliveira GP et al (2017) Filling gaps about mimivirus entry, uncoating and morphogenesis. J Virol. https://doi.org/10.1128/jvi.01335-17

21. Suhre K, Audic S, Claverie J-M (2005) Mimivirus gene promoters exhibit an unprecedented conservation among all eukaryotes. Proc Natl Acad Sci USA 102:14689-14693. https://doi.org/10.1073/ pnas.0506465102

22. Abrahão JS, Araújo R, Colson P, La Scola B (2017) The analysis of translation-related gene set boosts debates around origin and evolution of mimiviruses. PLoS Genet 13:e1006532. https://doi. org/10.1371/journal.pgen.1006532

23. Colson P, De Lamballerie X, Fournous G, Raoult D (2012) Reclassification of giant viruses composing a fourth domain of life in the new order Megavirales. Intervirology 55:321-332. https ://doi.org/10.1159/000336562

24. Moreira D, López-García P (2015) Evolution of viruses and cells: do we need a fourth domain of life to explain the origin of eukaryotes? Philos Trans R Soc Lond B Biol Sci 370:20140327. https:// doi.org/10.1098/rstb.2014.0327 
25. Gallot-Lavallée L, Blanc G, Claverie J-M (2017) Comparative genomics of Chrysochromulina ericina virus and other microalgainfecting large DNA viruses highlights their intricate evolutionary relationship with the established mimiviridae family. J Virol 91:JVI-00230. https://doi.org/10.1128/jvi.00230-17

26. Darling ACE, Mau B, Blattner FR, Perna NT (2004) Mauve: Multiple alignment of conserved genomic sequence with rearrangements. Genome Res 14:1394-1403. https://doi.org/10.1101/ gr.2289704
27. Edgar RC (2004) MUSCLE: a multiple sequence alignment method with reduced time and space complexity. BMC Bioinf 5:113. https://doi.org/10.1186/1471-2105-5-113

28. Tamura K, Stecher G, Peterson D et al (2013) MEGA6: molecular evolutionary genetics analysis version 6.0. Mol Biol Evol 30:2725-2729. https://doi.org/10.1093/molbev/mst197 